\title{
Anesthetic Care During Posterior Spinal Fusion in an Adolescent With Ebstein's Anomaly
}

\author{
Kenneth Phi ${ }^{\mathrm{a}, \mathrm{e}}$, David P. Martin ${ }^{\mathrm{b}, \mathrm{c}}$, Allan Beebe ${ }^{\mathrm{d}}$, \\ Jan Klamar ${ }^{d}$, Joseph D. Tobias ${ }^{b, c}$
}

\begin{abstract}
Ebstein's anomaly is a rare form of cyanotic congenital heart disease (CHD) that involves malformation and dysfunction of the tricuspid valve and right ventricle (RV). The severity of the defect impacts clinical presentation, survival, and treatment options. Presentation during the neonatal period with hypoxemia and cyanosis is noted in patients with severe tricuspid valve malformation, a hypoplastic RV, or RV outflow tract obstruction. However, presentation later in infancy is more common when there is a moderate tricuspid valve malformation and no associated RV outflow tract obstruction. Although Ebstein's anomaly is not generally associated with other congenital defects, patients may occasionally require surgery for other comorbid conditions. We describe the perioperative anesthetic management of an adolescent with Ebstein's anomaly for posterior spinal fusion. Previous reports of anesthetic care in this clinical scenario are reviewed, anesthetic considerations discussed, and options for intraoperative monitoring and anesthetic care presented.
\end{abstract}

Keywords: Ebstein's anomaly; Anesthesia; Congenital heart disease; Posterior spinal fusion

\section{Introduction}

Ebstein's anomaly is a rare form of cyanotic congenital heart disease (CHD) that involves malformation and dysfunction of the tricuspid valve and right ventricle (RV). It comprises less than $1 \%$ of all congenital heart defects [1]. It was first described by Wilhelm Ebstein in 1866 in a 19-year-old patient who presented with cyanosis, dyspnea, palpitations, and car-

Manuscript submitted March 5, 2020, accepted March 11, 2020

aThe Ohio State University School of Medicine, Columbus, OH, USA

${ }^{b}$ Department of Anesthesiology \& Pain Medicine, Nationwide Children's Hospital, Columbus, OH, USA

cDepartment of Anesthesiology \& Pain Medicine, The Ohio State University College of Medicine, Columbus, OH, USA

dDepartment of Orthopedic Surgery, Nationwide Children's Hospital and The Ohio State University, Columbus, OH, USA

${ }^{e}$ Corresponding Author: Kenneth Phi, The Ohio State University School of Medicine, Columbus, OH 43205, USA. Email: Kenneth.Phi@osumc.edu

doi: https://doi.org/10.14740/jmc3449 diomegaly $[2,3]$. Autopsy findings noted an extremely dilated right atrium, a patent foramen ovale (PFO), and an abnormal tricuspid valve where the proximal anterior leaflet was tethered to the ventricular endocardium. It was concluded that this cardiac anomaly consisted of a severe malformation of the tricuspid valve, absence of the Thebesian valve (which Ebstein saw as having no influence on the pathophysiology), and a PFO.

Subsequent to its original description, the anatomical variations of Ebstein's anomaly have been further defined into five major components: 1) Failure of delamination of the posterior and septal tricuspid valve leaflets leading to adherence to the ventricular myocardium; 2) Downward displacement of the tricuspid valve annulus; 3) Atrialization and dilation of a variable section of the RV; 4) Fenestrations, redundancy and tethering of the anterior leaflet; and 5) Dilation of the right atrioventricular (AV) junction $[4,5]$. In addition to the above pathology, global dilation of the RV can lead to compression of the left ventricular chamber due to displacement of the ventricular septum. The clinical features stem primarily from the RV dysfunction and right-to-left shunting if an atrial communication is present. Right-sided heart failure, cyanosis, and arrhythmias are common manifestations of Ebstein's anomaly.

Although the diagnosis is frequently made in utero due to the routine use of ultrasound screening during pregnancy, the severity of the defect impacts clinical presentation, survival, and treatment options. In particular, the clinical presentation depends on the severity of the tricuspid valve malformation and the associated RV compromise. Presentation with hypoxemia and cyanosis during the neonatal period occurs in patients with severe tricuspid valve malformation, a hypoplastic $\mathrm{RV}$, or RV outflow tract obstruction. Presentation later in infancy is more common in children with moderate tricuspid valve malformation and no associated RV outflow tract obstruction. Delayed presentation to adolescence and even adulthood may occur when an incidental murmur is heard or during a workup for arrhythmias [6].

Unlike other forms of CHD, Ebstein's anomaly is not generally associated with other congenital defects. However, patients may occasionally require surgery for other comorbid conditions. We describe the perioperative anesthetic management of an adolescent with Ebstein's anomaly for posterior spinal fusion (PSF). Previous reports of anesthetic care in this clinical scenario are reviewed, anesthetic considerations discussed, and options for intraoperative monitoring and anesthetic care presented. 


\section{Case Report}

Preparation of this case report followed the guidelines of the Institutional Review of Nationwide Children's Hospital (Columbus, $\mathrm{OH}, \mathrm{USA})$. The patient was a 14 -year-old, $31.5 \mathrm{~kg}$ adolescent who presented for PSF for treatment of neuromuscular scoliosis. Her past medical history was significant for CHD including Ebstein's anomaly of the tricuspid valve, bicuspid aortic valve, coarctation of the aorta, and subaortic valve stenosis. Additional medical concerns included a history of supraventricular tachycardia, cerebral palsy, gastroesophageal reflux disease, hypertension (renal), nephrocalcinosis, dysphagia, tracheostomy dependence, seizure disorder, vesicoureteral reflux (VUR), and asthma. Past surgical history included gastrostomy tube placement, adenoidectomy, bladder repair for VUR, tracheostomy, ureteral reimplantation, tympanostomy tube insertion, and repair of aortic coarctation and subvalvular stenosis. Current medications included omeprazole, baclofen (20 mg, one tablet three times daily), potassium citrate (three times daily), sennosides ( $5 \mathrm{~mL}$, once daily), norethindrone-ethinyl estradiol (one tablet daily), albuterol (90 $\mu \mathrm{g}$, two puffs every $4 \mathrm{~h}$ as needed), fluticasone (44 $\mu \mathrm{g}$, two puffs twice daily), sucralfate ( $1 \mathrm{~g}$, twice daily), nitrofurantoin (50 mg, once daily), lamotrigine (200 mg, twice daily), levetiracetam (1,000 mg, twice daily), and polyethylene glycol (17 g, once daily). Allergies included promethazine and sulfonamide antibiotics. Preoperative vitals revealed a heart rate of 102 beats/min, a respiratory rate of 20 breaths $/ \mathrm{min}$, blood pressure of 128/90 $\mathrm{mm} \mathrm{Hg}$, and an oxygen saturation of $100 \%$ on room air. The preoperative echocardiogram demonstrated the Ebstein's anomaly of the tricuspid valve, aortic coarctation status post resection with end-to-end anastomosis, bicuspid aortic valve, mild subvalvular and valvular aortic stenosis, severe right atrial and right ventricular enlargement related to the large atrialized portion of the $\mathrm{RV}$, mild tricuspid regurgitation and qualitatively normal biventricular systolic function. Physical examination revealed a thin adolescent with mild spasticity and joint contractures. Preoperative labs demonstrated a prothrombin time of $13.6 \mathrm{~s}$, international normalized ratio (INR) of 1.02, and activated partial thromboplastin time (aPTT) of 29 s. Renal function was unremarkable with blood urea nitrogen (BUN) of $7 \mathrm{mg} / \mathrm{dL}$ and creatinine of $0.38 \mathrm{mg} / \mathrm{dL}$. Hemoglobin was $13.1 \mathrm{~g} / \mathrm{dL}$ and hematocrit was $40.0 \%$. The patient was assigned an American Society of Anesthesiologists' classification 4 with a plan for general anesthesia and invasive hemodynamic monitoring. Special concerns included a history of difficult vascular access, the need for invasive monitoring, administration of blood products, and possible postoperative mechanical ventilation with planned intensive care unit (ICU) admission. The anesthetic plan, risks, benefits and alternatives were discussed with the parent and informed consent was obtained. The patient was held nil per os for $8 \mathrm{~h}$ prior to surgery. Upon arrival to the operating room, routine American Society of Anesthesiologists' monitors were placed. Additional monitoring included the use of a bispectral index and near infrared spectroscopy (NIRS) to monitor cerebral and tissue oxygenation. Anesthesia was induced by the inhalation of sevoflurane in nitrous oxide and oxygen via her tracheostomy. A 22-gauge peripheral intravenous cannula was placed followed by the administration of fentanyl $(50 \mu \mathrm{g})$ and rocuronium $(20 \mathrm{mg})$ to provide neuromuscular blockade. Two peripheral IV cannulas (18 gauge) and a radial arterial cannula were placed using ultrasound guidance. The tracheostomy was replaced with a 4.5 cuffed reinforced endotracheal tube (ETT). To allow for monitoring of somatosensory and motor evoked potentials, maintenance anesthesia included desflurane (expired concentration $2-4 \%$ ) in air/oxygen and a remifentanil infusion with a bolus dose of methadone [7]. In order to prevent fibrinolysis and limit blood loss, tranexamic acid was administered $(50 \mathrm{mg} / \mathrm{kg}$ followed by an infusion at $5 \mathrm{mg} / \mathrm{kg} / \mathrm{h}$ ). Intraoperative antibiotic therapy included cefazolin and gentamicin. A clevidipine infusion was administered to maintain the mean arterial pressure at $55-65 \mathrm{~mm} \mathrm{Hg}$ and limit blood loss. The surgical procedure lasted for approximately $7 \mathrm{~h}$. Intraoperative fluids consisted of $1,500 \mathrm{~mL}$ of Normosol-R ${ }^{\circledR}, 500 \mathrm{~mL}$ of $5 \%$ albumin, one unit of packed red blood cells (RBCs), and $205 \mathrm{~mL}$ of cell saver blood. Estimated blood loss for the procedure was 500 $\mathrm{mL}$. Following completion of the surgery, the reinforced ETT was removed and the tracheostomy replaced. The patient was transported to the post-anesthesia care unit (PACU) and then to the pediatric intensive care unit (PICU) for further monitoring and recovery. Postoperative pain control was achieved with acetaminophen every $6 \mathrm{~h}$ alternating with ketorolac every $6 \mathrm{~h}$, gabapentin (three times a day), valium as needed, and hydromorphone delivered via patient/nurse controlled anesthesia. Postoperative prophylaxis for deep venous thrombosis (DVT) included the use of sequential compression devices and daily subcutaneous enoxaparin sodium. Her routine home medications were restarted postoperatively. Her postoperative course was complicated by a multi-drug resistant urinary tract infection due to Escherichia coli, which was treated with meropenem. Additionally, she developed a new $\mathrm{S}_{3}$ that was initially thought to be due to fluid overload, but after a negative workup and no improvement with diuresis therapy, was confirmed to be a mitral click. She also required transfusion of one unit of packed RBCs due to anemia. She was discharged home on postoperative day 17 in stable condition and is doing well on follow-up visits to the orthopedic clinic.

\section{Discussion}

As with any anesthetic encounter, effective perioperative care begins with a preoperative evaluation with a medication review, identification of the end-organ impact of the primary disease process, review of associated comorbid conditions, and a consideration of the implications of the intended surgical procedure. In general, the anesthetic implications of Ebstein's anomaly include right-to-left shunting with the risk of paradoxical emboli, pooling of blood in the right atrium, arrhythmias, the potential for increased pulmonary vascular resistance (PVR), and right ventricular dysfunction (Table 1). All of these perioperative issues are rooted in the anatomic abnormalities of Ebstein's anomaly, specifically the atrialization of the RV and the abnormal morphology of the tricuspid valve [4]. In general, CHD with a right-to-left intracardiac shunt results in 
Table 1. Perioperative Cardiac Implications of Ebstein's Anomaly

Risk for arrhythmias
Right-to-left shunting at the atrial level
Risk of paradoxical emboli
Shunt with hypoxemia
Pooling and recirculation of blood in the right atrium
$\quad$ Decreased onset of action of intravenously administered medications
Potential for increased pulmonary vascular resistance
Right ventricular dysfunction
Left ventricular dysfunction due to RV dilation with displacement of the ventricular septum with compression of the left ventricular chamber
and impairment of ventricular filling

$\mathrm{RV}$ : right ventricle.

the prolongation of inhalation induction, while intravenous induction is faster $[8,9]$. However, the unusual anatomy of Ebstein's anomaly can lead to pooling and recirculation of blood in the right atrium with the delayed delivery of medications to the periphery and hence a delayed onset of action of intravenous anesthetic agents and neuromuscular blocking agents.

Right-to-left shunting may also predispose patients to paradoxical emboli during the perioperative period and the risk of systemic complications including stroke or brain abscess $[10,11]$. The potential impact of this issue is illustrated by anecdotal case reports. Melao et al reported recurrent transient ischemic attacks in an adult with Ebstein's anomaly and a PFO with a history of intravenous drug abuse [10]. Computed tomography (CT) imaging revealed several foci of ischemic infarctions that were attributed to paradoxical emboli. Lonnebakken et al reported the development of a brain abscess related to right-to-left shunting in a patient with Ebstein's anomaly [11]. Given these concerns, intraoperative attention should be directed at ensuring that all intravenous infusions are free of air and consideration given to the use of filters on intravenous infusions to prevent the inadvertent administration of air or particulate matter. Given the presence of right-to-left shunting, these small volumes of air or particulate matter may not be cleared by the pulmonary circulation. The potential for emboli and the degree of hypoxemia are greater with increases in PVR which may be pre-existing or exacerbated by intraoperative care (see below). Postoperatively, prophylactic measures should be instituted to prevent the formation of deep venous thrombi especially when prolonged bedrest is necessary [12]. In our patient, postoperative DVT prophylaxis included the use of sequential compression devices on the lower extremities and daily subcutaneous enoxaparin sodium until she was fully ambulatory.

Depending on the magnitude of the defect, RV function may be compromised [13]. Preoperative echocardiography is indicated to evaluate the degree of RV dysfunction, assess left ventricle (LV) function, assess for the presence and severity of pulmonary hypertension, and determine the presence of other associated CHD. Associated CHD most commonly includes an atrial septal defect (ASD), a patent ductus arteriosus (PDA), and abnormalities of the pulmonary valve. Kumar et al reported that 18 of 55 patients with Ebstein's anomaly had an associated CHD [14]. Our patient had a history of a bicuspid aortic valve, an aortic coarctation that has required surgical intervention as well as valvular and sub-valvular aortic stenosis that was demonstrated on the preoperative echocardiogram. Their magnitude was not such that intervention was deemed necessary at this time.

Associated congenital heart defects may have either a beneficial or detrimental effect on function and the patient's hemodynamic status. An unrepaired ASD can lead to excessive right-to-left shunting and cyanosis, which is worsened by pulmonary valve abnormalities whether it is anatomical atresia or functional stenosis [13]. A PDA may be essential as it maintains pulmonary blood flow in the setting of a dysfunctional $\mathrm{RV}$ with poor right ventricular output in patients with severe Ebstein's anomaly. During the neonatal period, prostaglandin $\mathrm{E}$ (PGE) administration may be necessary to maintain patency of the PDA and provide pulmonary blood flow until surgical intervention.

Left heart function can also be affected by Ebstein's anomaly as severe RV dilatation can impair filling and function of the LV due to displacement of the interventricular septum [15]. The reduction in LV filling and ejection fraction is mostly noted in diastole as a result of the leftward displacement of the interventricular septum. The worst outcomes due to this phenomenon are expected in individuals with severe Ebstein's who have a severely malformed and atrialized RV. Once again, these issues can be assessed during the preoperative echocardiogram. Our patient had normal biventricular function and no evidence of intraventricular bowing or compromise of LV filling. If depressed function is noted, adequate preload should be maintained and anesthetic agents with negative inotropic and vasodilatory effects (barbiturates, propofol) should be avoided. Etomidate may be considered for anesthetic induction given its negligible effects on myocardial performance and hemodynamic function [16]. Furthermore, central venous access for the measurement of central venous pressure and the administration of vasoactive agents as well as invasive hemodynamic monitoring with arterial access should be considered. The latter will allow for early identification and immediate treatment of hemodynamic compromise. As demonstrated by our patient, we have found that NIRS may be a valuable adjunct to intraoperative monitoring $[17,18]$. Using optical technology 
similar to pulse oximetry and based on the relative absorption of infrared light by different hemoglobin species, the monitor generates a measurement of regional tissue (somatic and cerebral) oxygen saturation and therefore allows a continuous assessment of the adequacy of cardiac output and tissue oxygen delivery.

Individuals with Ebstein's anomaly have a proportionally higher risk of arrhythmias as a result of the abnormal anatomy of the right heart. Various intrinsic arrhythmias such as accessory AV pathways, AV nodal re-entry, and atriofasicular fibers have been reported in patients with Ebstein's anomaly. In addition, extrinsic arrhythmias such as atrial macro re-entry and focal atrial tachycardia may also occur [19]. Of the arrhythmias seen in conjunction with Ebstein's, accessory pathway (AP) is the most common and has a higher incidence when compared to patients with a structurally normal heart [20]. This pathway is thought to originate from the posterior and septal area of the atrialized RV. The abnormal pathways are often different than individuals with AP arrhythmias and normal structural hearts which makes curative ablations more difficult. Identifying the presence of any pre-existing arrhythmias in patients with Ebstein's anomaly is crucial in preparation for surgery. Standard 12-lead electrocardiograms and Holter monitoring may be helpful in identifying preoperative arrhythmias, especially those that are asymptomatic at baseline. Initiating antiarrhythmic therapy and continuing it during the perioperative period is essential.

Patients with Ebstein's anomaly may have pre-existing pulmonary hypertension and exacerbations or alterations may occur intraoperatively related to the anesthetic technique or surgical manipulation. Identifying patients with elevated PVR or pulmonary arterial hypertension (PAH) in the preoperative period is essential in this high risk population [21]. Intraoperative anesthetic care is directed at the regulation or avoidance of factors that modulate or increase PVR including hypoxemia, alveolar hypoxia, metabolic acidosis, hypercarbia, and sympathetic nervous system activation from inadequate analgesia [22]. Avoidance or limitation of agents with direct negative inotropic or vasodilatory effects such as propofol or barbiturates is suggested along with careful titration of the volatile anesthetic agents. Preoperative planning is essential with the development of a strategy to manage an intraoperative pulmonary hypertensive crisis. In such, identification of the underlying cause is essential with reversal of the inciting event if discovered. Other intraoperative management techniques to treat increased PVR may include the administration of pulmonary vasodilators including inhaled nitric oxide, phosphodiesterase inhibitors, or prostacyclin analogues.

When planning an anesthetic technique, there must be consideration of the implications of the intended surgical procedure. In our patient, intraoperative monitoring of motor and somatosensory evoked potentials was planned and facilitated by our usual practice of desflurane (expired concentration $2-4 \%$ ) in air/oxygen and a remifentanil infusion preceded by an intraoperative dose of methadone to provide postoperative analgesia. The technique not only allows for effective neurophysiological monitoring, but also allows for a rapid intraoperative wakeup test if there are changes in neurophysiological monitoring. A rapid emergence is also feasible with limited residual effects of the anesthetic agents on airway and respiratory function. Given the potential for blood loss, arterial access and adequate peripheral venous access were obtained. Due to our patient's status and the previous history of difficulties with vascular access, ultrasound guidance was used to facilitate placement of arterial and peripheral venous cannulas [23].

Spinal surgery including PSF may result in significant intraoperative blood loss, at times in excess of an entire blood volume. This may be exacerbated by the presence of abnormalities of coagulation function in patients with cyanotic CHD [24]. Preoperative coagulation function should be evaluated and treatment with vitamin $\mathrm{K}$ or blood products should be given as needed. Multiple techniques to minimize intraoperative blood loss and allogeneic transfusions can be employed during major orthopedic procedures including acute normovolemic hemodilution, intraoperative blood salvage, controlled hypotension, and manipulation of the coagulation cascade with antifibrinolytic agents (epsilon-aminocaproic acid, tranexamic acid) [25]. In our clinical practice, the commonly used techniques include blood pressure management, tranexamic acid as an anti-fibrinolytic agent, and intraoperative cell salvage.

In summary, we present the perioperative considerations of a patient with congenital Ebstein's anomaly. Perioperative considerations include right-to-left shunting with the potential for paradoxical emboli, pooling and recirculation of blood in the right atrium with prolonged onset time of intravenous medications, increased PVR and perioperative pulmonary hypertension, right and left ventricular dysfunction, and the potential for arrhythmias. Preoperative evaluation using echocardiography and electrocardiography can help identify underlying rhythm, function or structural abnormalities that may predispose to perioperative complications. Key cardiovascular goals include maintenance of normal sinus rhythm, adequate preload, avoidance of significant changes in systemic vascular resistance (SVR) or PVR, and avoidance of medications and factors that depress myocardial function. Prompt identification of hemodynamic instability or inadequate tissue perfusion with appropriate correction will lead to improved outcomes.

\section{Acknowledgments}

None to declare.

\section{Financial Disclosure}

None to declare.

\section{Conflict of Interest}

None to declare.

\section{Informed Consent}

In accordance with IRB of Nationwide Children's Hospital, 
need for documentation of informed consent is waved.

\section{Author Contributions}

KP prepared first and subsequent drafts; JDT contributed to the concept and review of all drafts; DPM contributed to intraoperative care, review of initial and subsequent drafts; $A B$ and $\mathrm{JK}$ contributed to intraoperative care and review of final manuscript.

\section{Data Availability}

Any inquiries regarding supporting data availability of this study should be directed to the corresponding author.

\section{References}

1. Yuan SM. Ebstein's anomaly: genetics, clinical manifestations, and management. Pediatr Neonatol. 2017;58(3):211-215.

2. van Son JA, Konstantinov IE, Zimmermann V. Wilhelm Ebstein and Ebstein's malformation. Eur J Cardiothorac Surg. 2001;20(5):1082-1085.

3. Mann RJ, Lie JT. The life story of Wilhelm Ebstein (18361912) and his almost overlooked description of a congenital heart disease. Mayo Clin Proc. 1979;54(3):197-204.

4. Edwards WD. Embryology and pathologic features of Ebstein's anomaly. Prog Pediatr Cardiol. 1993;2:5-15.

5. Radford DJ, Graff RF, Neilson GH. Diagnosis and natural history of Ebstein's anomaly. Br Heart J. 1985;54(5):517522.

6. Celermajer DS, Bull C, Till JA, Cullen S, Vassillikos VP, Sullivan ID, Allan L, et al. Ebstein's anomaly: presentation and outcome from fetus to adult. J Am Coll Cardiol. 1994;23(1):170-176.

7. Martin DP, Bhalla T, Thung A, Rice J, Beebe A, Samora W, Klamar J, et al. A preliminary study of volatile agents or total intravenous anesthesia for neurophysiological monitoring during posterior spinal fusion in adolescents with idiopathic scoliosis. Spine (Phila Pa 1976). 2014;39(22):E1318-1324.

8. Cannesson M, Piriou V, Neidecker J, Lehot JJ. [Anaesthesia for non cardiac surgery in patients with grownup congenital heart disease]. Ann Fr Anesth Reanim. 2007;26(11):931-942.

9. Chowdhury D. Pathophysiology of congenital heart diseases. Ann Card Anaesth. 2007;10(1):19-26.

10. Melao F, Correia AS, Maciel MJ. Paradoxical embolism associated with Ebstein's anomaly in an adult: case report.
Rev Port Cardiol. 2013;32(12):1023-1025.

11. Lonnebakken MT, Greve G, Leirgul E, Gerdts E. Brain abscess caused by paradoxical embolization in Ebstein's anomaly. Eur J Echocardiogr. 2008;9(1):105-106.

12. Boonyawat K, Crowther MA. Venous thromboembolism prophylaxis in critically ill patients. Semin Thromb Hemost. 2015;41(1):68-74.

13. Knott-Craig CJ, Goldberg SP, Ballweg JA, Boston US. Surgical decision making in neonatal Ebstein's anomaly: an algorithmic approach based on 48 consecutive neonates. World J Pediatr Congenit Heart Surg. 2012;3(1):1620.

14. Kumar AE, Fyler DC, Miettinen OS, Nadas AS. Ebstein's anomaly. Clinical profile and natural history. Am J Cardiol. 1971;28(1):84-95.

15. Benson LN, Child JS, Schwaiger M, Perloff JK, Schelbert HR. Left ventricular geometry and function in adults with Ebstein's anomaly of the tricuspid valve. Circulation. 1987;75(2):353-359.

16. Tobias JD. Etomidate: applications in pediatric anesthesia and critical care. J Intensive Care Med. 1997;12:324-326.

17. Tobias JD. Cerebral oxygenation monitoring: nearinfrared spectroscopy. Expert Rev Med Devices. 2006;3(2):235-243.

18. Dewhirst E, Hakim M, Tobias JD. Additional applications of intraoperative cerebral oxygenation monitoring using near infrared spectroscopy. Pediatr Anesth Crit Care J. 2016;4:18-23.

19. Walsh EP. Ebstein's Anomaly of the Tricuspid Valve: A Natural Laboratory for Re-Entrant Tachycardias. JACC Clin Electrophysiol. 2018;4(10):1271-1288.

20. Saul JP, Walsh EP, Triedman JK. Mechanisms and therapy of complex arrhythmias in pediatric patients. J Cardiovasc Electrophysiol. 1995;6(12):1129-1148.

21. Carmosino MJ, Friesen RH, Doran A, Ivy DD. Perioperative complications in children with pulmonary hypertension undergoing noncardiac surgery or cardiac catheterization. Anesth Analg. 2007;104(3):521-527.

22. Friesen RH, Williams GD. Anesthetic management of children with pulmonary arterial hypertension. Paediatr Anaesth. 2008;18(3):208-216.

23. Tobias JD, Martin DP, Bhalla T. Ultrasound-guided peripheral venous and arterial cannulation in the pediatric population. Anaesth Pain Intensive Care. 2015;19:311316.

24. van Nieuwenhuizen RC, Peters M, Lubbers LJ, Trip MD, Tijssen JG, Mulder BJ. Abnormalities in liver function and coagulation profile following the Fontan procedure. Heart. 1999;82(1):40-46.

25. Tobias JD. Strategies for minimizing blood loss in orthopedic surgery. Semin Hematol. 2004;41(1 Suppl 1):145156. 